HNO 2009 · 57:1218-1220

DOI 10.1007/s00106-009-2021-1

Online publiziert: 20. November 2009

(c) Springer Medizin Verlag 2009

\author{
N. Stasche \\ Westpfalz-Klinikum, Kaiserslautern
}

\title{
Endoskopie in der HNO
}

körper und nur noch 35\% der Ösophagusfremdkörper durch HNO-Ärzte endoskopisch entfernt werden. Die Mehrzahl der Fremdkörper wird heute flexibel bronchoskopisch und flexibel ösophagoskopisch von anderen Fachrichtungen entfernt.

Auf der anderen Seite werden auch heute noch mehr als 90\% der starren Ösophagoskopien in Deutschland in der HNO durchgeführt. Es ist wichtig zu erwähnen, dass in bestimmten Situationen z. B. bei eingeklemmten Fremdkörpern in der oberen Ösophagusenge oder bei der sicheren Identifikation von Tumoren im hypopharyngoösophagealen Übergang, nur das starre Rohr zielführend einzusetzen ist. Hauptindikation von Tracheobronchoskopie und Ösophagoskopie in der HNO ist nach wie vor das Staging von Kopf-Hals-Tumoren im Rahmen der Panendoskopie. Die frühzeitige Entdeckung von synchronen oder metachronen Zweitkarzinomen verbessert die Prognose dieser Patienten nachhaltig [2].

Neue Entwicklungen in der Gerätetechnik, des Zubehörs und bei den flexiblen Endoskopen haben die flexiblen endoskopischen Diagnose- und Interventionsmöglichkeiten erheblich vorangebracht. Besonders in der gastroenterologischen Endoskopie sind diese Verbesserungen in die Praxis umgesetzt worden.

Die ungünstige Prognose und die ständige Zunahme von malignen Tumoren im Gastrointestinaltrakt haben in den letzten Jahren das Interesse auf die Früherkennung solcher Tumoren gelenkt. Durch moderne hochauflösende flexible Videoendoskope und den zusätzlichen Einsatz von Vitalfärbemethoden hat sich die Detailerkennbarkeit der endoskopisch untersuchten Schleimhäute wesentlich verbessert. Die Chromoendoskopie ist heute fester Bestandteil der gastroenterologischen
Endoskopie und hat durch die Möglichkeit, auch intraepitheliale Neoplasien zu entdecken, zu einer deutlichen Verbesserung der Früherkennung geführt [3].

\section{(2) Die Chromoendoskopie ist heute fester Bestandteil der gastroenterologischen Endoskopie}

Auch neue Entwicklungen in der Farblichtgebung und Bildverarbeitung bei den flexiblen Endoskopen erlauben eine bessere Visualisierung von Gefäßund Schleimhautstrukturen. Die Technik des "narrow band imaging“ erlaubt es so, schon Frühläsionen an den Schleimhäuten des oberen Gastrointestinaltrakts zu erkennen [4].

Hierzu gehört auch die Einführung der konfokalen Laser-Endomikroskopie in die Praxis. Diese Untersuchungstechnik erlaubt auf molekularer Ebene eine Beurteilung nicht nur oberflächlicher Schleimhautareale, sondern auch darunter liegender Schichten, sodass endoskopische „Live-Bilder" resultieren, wie sie der Pathologe üblicherweise auf seinen histologischen Schnittbildern sieht $[5,6]$. Unter anderem beschäftigt sich jetzt auch in der HNO eine Arbeitsgruppe in Rostock mit dem Einsatz der konfokalen Laser-Endomikroskopie in der Laryngoskopie [7].

In Verbindung mit vor allem in Japan entwickelten neuen endoskopischen Resektionstechniken (EMR, „endoscopic mucosal resection") lassen sich heute umschriebene, oberflächliche Frühneoplasien im Gastrointestinaltrakt sicher kurativ entfernen. Die Erfolge der neuen flexiblen Diagnosetechniken haben zur Einführung einer flächendeckenden Koloskopie in das gesetzliche Krebsfrüherkennungsprogramm in Deutschland im Jahr 2002 geführt, von der es aktuell eine ers- 
te evidenzbasierte Auswertung gibt [8]. Nach der Analyse von 1,8 Mio. Koloskopien wird in Deutschland bis 2010 mit einer Verhinderung von etwa 15.000 kolorektalen Karzinomen gerechnet. Von der Einführung eines solchen Vorsorgeprogramms in der HNO, z. B. zur Früherkennung von Kopf-Hals-Tumoren, sind wir in Deutschland noch weit entfernt. Risikogruppen sind eindeutig identifizierbar. Vor diesem Hintergrund ist eine konsequente Suche nach Zweitkarzinomen im Kopf-Hals-Bereich bei manifesten Ösophagus- oder Bronchialkarzinomen durch den HNO-Arzt genauso zu fordern wie die endoskopische Abklärung von Ösophagus und Tracheobronchialbaum bei Patienten mit Kopf-Hals-Tumoren.

NOTES ist ein neues spannendes Konzept, welches das Potenzial hat, die interventionelle Endoskopie und die minimalinvasive Chirurgie zu revolutionieren. Der Begriff NOTES („natural orifice transluminal endoscopic surgery") wurde 2006 in den USA von Gastroenterologen und Chirurgen geprägt $[9,10,11]$. Er beschreibt Techniken und Eingriffe, bei denen über natürliche Körperöffnungen wie Mund, Anus, Vagina oder Harnröhre ein Zugang zur Bauchhöhle geschaffen wird. Die endoskopische Chirurgie über natürliche Körperöffnungen ist zunächst wegen der Zunahme der Zahl von Patienten mit extremer Obesitas befördert worden. Die fehlende Möglichkeit von Zugangswegen der minimalinvasiven Chirurgie (MIC) durch die Bauchdecke von extrem adipösen Patienten z. B. zur Cholezystektomie und das extrem hohe perioperative Risiko dieser Patienten führte zur Suche nach neuen, auch unkonventionellen Wegen in der endoskopischen Chirurgie. In der Gynäkologie wurde bereits seit längerem der transvaginale Zugang auch für verschiedene Eingriffe im Bauchraum bis hin zur Appendektomie eingesetzt.

Gastroenterologen und Chirurgen haben mit zunehmender Verbesserung der flexiblen endoskopischen Technik und Instrumente in den 1990er-Jahren den transgastralen und transrektalen Weg zum Pankreas, zur Gallenblase und zur Milz gesucht, um an diesen Organen und benachbarten Räumen und Strukturen mit endoskopischen Mitteln zu intervenieren. Diese Entwicklungen laufen der-

\title{
Hier steht eine Anzeige.
}

\author{
算 Springer
}


zeit parallel in den chirurgischen Fächern und in der Gastroenterologie ab. Sie haben zur Gründung von entsprechenden Arbeitsgruppen in den USA und Europa geführt. Das US-amerikanische Konsortium trägt den Namen NOSCAR, d. h. Natural Orifice Surgery Consortium for Assessment and Research (www.noscar.org). In den Arbeitsgruppen wird der Grundstein für eine vor allem patientensichere Einführung dieser neuen Technologie gelegt. Neben der Entwicklung gänzlich neuer Zugangswege wird ein extremer Innovationschub bei der Entwicklung neuer Endoskope, Instrumente und Nahttechniken erwartet. All diese Entwicklungen beschreibt und wertet Jürgen Hochberger, Gastroenterologe aus Hildesheim, Mitglied der D-NOTES-Arbeitsgruppe, in seinem Übersichtsartikel.

In der Kopf-Hals-Chirurgie finden diese Innovationen derzeit in der Entwicklung immer neuer Robotik- und Navigationssysteme und ganz aktuell in der Entwicklung der transoralen endoskopischen Halschirurgie statt. Diese Entwicklungen stehen erst am Anfang und haben neue Zugangswege zu den Halsorganen zum Ziel. Beschrieben sind solche Ansätze bei der videoassistierten und transoralen Schilddrüsenchirurgie [12, 13, 14], bei der Sentinel-node-Diagnostik [14] und beim transoralen Zugang zur Glandula submandibularis [15].

Ingo F. Herrmann, Rom, hat sich seit Jahren der funktionellen Endoskopie des Schluckvorgangs, der gastroösophagealen Refluxerkrankung und der extraösophagealen Refluxmanifestationen im HNOFach verschrieben. In seinem Übersichtsartikel beschreibt er aktuelle Erkenntnisse auf diesem Gebiet. Er konnte zeigen, wie die einzelnen Schritte des Schluckvorgangs miteinander verknüpft sind, und hat daraus neue Erkenntnisse für die Behandlung refluxassoziierter HNO-Symptome und Befunde abgeleitet.

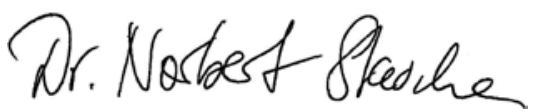

N. Stasche

\section{Korrespondenzadresse}

\section{Prof. Dr. N. Stasche}

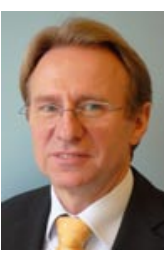

Westpfalz-Klinikum

Hellmut-Hartert-Straße 1 67655 Kaiserslautern nstasche@westpfalzklinikum.de

Interessenkonflikt. Der korrespondierende Autor gibt an, dass kein Interessenkonflikt besteht.

\section{Literatur}

1. Schmidt H, Stasche N, Keller A, Hörmann K (2009) Tracheobronchoscopy and esophagoscopy in current ear-nose-throat practice: an update. Eur Arch Otorhinolaryngol. DOI 10.1007/s00405-009-0997-5

2. Scherübl H, Steinberg J, Schwertner C, Mir-Salim P et al (2008) „Field cancerization" im oberen Aerodigestivtrakt: Überwachungsempfehlungen für Risikopersonen. HNO 56:603-608. DOI 10.1007/ s00106-007-1616-7

3. Connor MJ, Sharma P (2004) Chromoendoscopy and magnification endoscopy for diagnosing esophageal cancer and dysplasia. Thorac Surg Clin 14:87-94

4. Takenaka R, Kawahara Y, Okada H, Hori K et al. (2009) Narrow-band imaging provides reliable screening for esophageal malignancy in patients with head and neck cancers. Am J Gastroenterol [Epub ahead of print]

5. Dunbar K, Canto M (2008) Confocal endomicroscopy. Curr Opin Gastroenterol 24:631-637

6. Kiesslich R, Goetz M, Neurath MF (2008) Virtual histology. Best Pract Res Clin Gastroenterol 22:883-897

7. Just T, Stave J, Kreutzer HJ, Guthoff R, Pau HW (2007) Confocal microscopic evaluation of epithelia of the larynx. Laryngorhinootologie 86:644-648

8. Brenner $\mathrm{H}$, Hoffmeister M, Brenner G, Altenhofen L, Haug U (2009) Expected reduction of colorectal cancer incidence within 8 years after introduction of the German screening colonoscopy programme: Estimates based on 1,875,708 screening colonoscopies. Eur J Cancer 45:2027-2033

9. Kalloo AN, Singh VK, Jagannath SB et al (2004) Flexible transgastric peritoneoscopy: a novel approach to diagnostic and therapeutic interventions in the peritoneal cavity. Gastrointest Endosc 60: 114-147

10. Kantsevoy SV,Hu B, Jagannath SB et al (2006) Transgastric endoscopic splenectomy: is it possible? Surg Endosc 20:522-525

11. Swain P (2008) Nephrectomy and natural orifice translumenal endoscopy (NOTES): transvaginal, transgastric, transrectal, and transvesical approaches. J Endourol 22:811-818

12. Tan CT, Cheah WK, Delbridge L (2008) "Scarless" (in the neck) endoscopic thyroidectomy (SET): an evidence-based review of published techniques. World J Surg 32:1349-1357

13. Benhidjeb T, Wilhelm T, Harlaar J, Kleinrensink GJ et al (2009) Natural orifice surgery on thyroid gland: totally transoral video-assisted thyroidectomy (TOVAT): report of first experimental results of a new surgical method. Surg Endosc 23:1119-1120

14. Werner JA, Sapundzhiev NR, Teymoortash A, Dünne AA et al (2004) Endoscopic sentinel lymphadenectomy as a new diagnostic approach in the N0 neck. Eur Arch Otorhinolaryngol 261:463-468

15. Kauffman RM, Netterville JL, Burkey BB (2009) Transoral excision of the submandibular gland: techniques and results of nine cases. Laryngoscope 119:502-507

\section{Lernen mit den Augen des Lehrers}

Die Entwickler der „EyeSeeCam“ haben den mit 10.000 Euro dotierten Preis beim RoboDays-Festival im dänischen Odense gewonnen. Damit würdigte die Jury dieses ganz besondere Kamerasystem, welches die Augenbewegung eines Menschen originalgetreu - also mit geringer Zeitverzögerung - wieder gibt. Entwickelt wurde dieses „künstliche Auge“ im Klinikum der Universität München.

Die EyeSeeCam wurde bereits im Operationssaal getestet. Mit einem Gestell am Kopf befestigt, hat die Kamera live und direkt alle Blicke des Arztes während eines Eingriffs mit einer Zeitverzögerung von nur zehn Millisekunden übertragen. Damit ist die Kamera derzeit das schnellste entsprechende System weltweit. Sozusagen mit den Augen des Chirurgen verfolgten die Zuschauer die Operation.

Künftig soll das System deshalb für Lehrzwecke genutzt werden, um beispielsweise Eingriffe aus dem Operationssaal in einen Hörsaal mit Medizinstudenten zu übertragen.

Nicht zuletzt soll die EyeSeeCam in der Medizin genutzt werden, um bei einem Eingriff die Arbeitsbelastung und die visuelle Aufmerksamkeitsverteilung etwa von Chirurgen oder Anästhesisten zu messen. Durch Analyse der Blickbewegungen lässt sich ermitteln, ob etwa ein Anästhesist die wichtigen Geräte auch in einer Notfall-Situation noch immer im Blickfeld hat und ob er sie sicher bedienen kann. Der Einsatz der EyeSeeCam würde so zur rechtzeitigen Erkennung einer Notfallsituation beitragen und langfristig die Patientensicherheit erhöhen.

Klinikum der Universität München (LMU), www.klinikum.uni-muenchen.de 